\title{
Endoscopic third ventriculostomy (ETV) for treatment of adult hydrocephalus: long-term followup with 163 patients
}

\author{
Albert Isaacs", Geberth Urbaneja, Mark Hamilton \\ From Hydrocephalus 2015 \\ Banff, Canada. 18-21 September 2015
}

\section{Introduction}

Treatment of specific patterns of symptomatic hydrocephalus in the adult patient may be accomplished with endoscopic third ventriculostomy (ETV) as an alternative to insertion of a ventriculoperitoneal (VP) shunt or when VP shunt failure occurs. Treatment of hydrocephalus with a VP shunt, while effective, is associated with a significant shunt failure rate that results in VP shunt revision surgery. This review examines a single center experience with ETV to treat hydrocephalus in symptomatic adult patients.

\section{Methods}

Adult patients $(>/=18$ years $)$ with a diagnosis of hydrocephalus who were treated with ETV in Calgary between January 1994 and July 2014 were reviewed using a clinic database and registry. All patients were treated by one neurosurgeon.

\section{Results}

163 adult patients with symptomatic hydrocephalus treated with ETV were identified (male=92; female $=71$ ). Mean age at the time of ETV was 46 years (range 18-83 years). 112 underwent ETV as a primary treatment and 51 patients underwent treatment after presenting with VP shunt failure (secondary ETV). 113/163 patients had a diagnosis of aqueductal stenosis, 22/163 had a diagnosis of tumor. Mean followup was 8.2 years (range 0.3-18.4 years). Symptoms in 149/163 (91.4\%) of ETV patients were better or unchanged at last followup. 104/118 (88.1\%) of primary ETV patients were shunt free at last

* Correspondence: akm.isaacs@gmail.com University of Calgary, Canada creativecommons.org/licenses/by/4.0), which permits unrestricted use, distribution, and reproduction in any medium, provided the original work is properly cited. The Creative Commons Public Domain Dedication waiver (http://creativecommons.org/publicdomain/ zero/1.0/) applies to the data made available in this article, unless otherwise stated. 